\title{
LA SITUACIÓN DE LOS SLURS
}

On the situation of slurs

\begin{abstract}
Sofia Checchi ${ }^{1}$
\section{RESUMEN}

La bibliografía reciente sobre slurs se enfrentó a un problema de clasificación. Díaz Legaspe (2018) ofreció una nueva taxonomía: "marginales" (out-group) y normalizantes. El primer subgrupo engloba los slurs que no dependen fuertemente del contexto y uso, correferentes con contrapartes neutras. Esto posibilita que no haya diferencias en términos de condiciones de verdad entre enunciados que contengan un slur y aquellos que incluyan su contraparte neutra. Los normalizantes se distinguen por su restricción referencial: su referencia es inferior cuantitativamente a la de su contraparte neutra, y esto impacta en sus condiciones de verdad. El presente trabajo dará una explicación de la restricción referencial desde la semántica situacional de Barwise y Perry. Esta teoría da cuenta de la sensibilidad contextual de los slurs normalizantes, en tanto la situación de referencia contiene las normas sociales vigentes. Los rasgos de la situación de emisión serán decisivos para determinar la situación focal, i. e., la referencia.
\end{abstract}

Palabras clave: Slurs. Referencia. Contexto. Semántica situacional.

\begin{abstract}
Recent bibliography on group pejoratives has faced a classification problem. Díaz Legaspe (2018) offered a new taxonomy: out-group and normalizing slurs. The first subgroup includes slurs that do not depend strongly on context or use and corefer with neutral. This guarantees that there are no differences concerning the truth conditions among statements that contain a slur and those which include its neutral counterpart. Normalizing slurs are distinguished by their referential restriction -the reference of these terms is quantitatively inferior to the one of their neutral counterparts, and this affects the truth conditions of these slurs. The present article provides an explanation to referential restriction from Barwise \& Perry's situational semantics. it is a proper model to account for the contextual sensitivity that normalizing slurs show, given that the reference situation contains the prevailing social norms. The features of the utterance situation will be decisive in order to determine the focal situation, i. e., the reference.
\end{abstract}

Keywords: Slurs. Reference. Context. Situation semantics.

\footnotetext{
${ }^{1}$ Universidad de Buenos Aires.

E-mail: sofia.checchi@hotmail.com. ORCID: 0000-0003-4262-9192.
} 


\section{Introducción}

En los últimos años, los términos peyorativos de grupo o slurs $^{2}$ han revestido un creciente interés dentro del campo de estudio de la filosofía del lenguaje. Cepollaro y Thommen (2019) describen el fenómeno de la siguiente manera:

Natural languages have certain pejorative predicates that target groups or individuals on the basis of properties such as their ethnicity, gender, sexual orientation, and the like. Utterances of such expressions typically evoke strong emotional reactions in bystanders and targets, whereas standard predicates usually do not. (CEPOLLARO; THOMMEN, 2019) ${ }^{3}$

Los análisis se han multiplicado y sofisticado en diferentes direcciones, atendiendo a aspectos semánticos, pragmáticos, sociales, y culturales. Frutos de estas investigaciones han sido dos consideraciones centrales en pos de aproximar el funcionamiento y carácter del objeto, a saber: i) el grado de impacto que supone el uso de un slur en las condiciones de verdad del enunciado que lo contiene, y ii) la identificación de tendencias en función de las particularidades del grupo al que está dirigido (o grupo target), la fuerza derogatoria, la ofensa, y demás factores que co-ocurren en el uso de un slur.

La primera de estas consideraciones ha dividido las aguas para quienes abordan este fenómeno desde una perspectiva semántica. Por un lado, una de las propuestas sostiene que la contribución que el uso de un slur hace a las condiciones de verdad de un enunciado es nula, ya que distingue, en el análisis, dos tipos de contenido asociados a ese término: uno puramente expresivo y una contraparte neutra, no peyorativa, que correfiere con el slur. Por otro lado, existen líneas de análisis que no abogan por esta separación de contenido, y mantienen que o bien hay razones por las cuales en determinados casos es difícil o imposible encontrar contrapartes neutras para los slurs, o bien analizan el fenómeno bajo una luz más vinculada al vértice so-

\footnotetext{
${ }^{2}$ Es menester advertir que a lo largo de este artículo se hará mención de términos peyorati vos en ejemplificaciones y estipulaciones teóricas, pero que la autora no respalda, aprueba, ni comparte las actitudes y creencias en las que se cimenta su uso y la representación de los grupos en cuestión.

3 "Los lenguajes naturales tienen ciertos predicados peyorativos que se dirigen a grupos o individuos en función de propiedades tales como su etnia, género, orientación sexual, y similares. Emisiones de tales expresiones típicamente evocan reacciones emocionales fuertes entre las personas presentes y sus objetivos, mientras que los predicados estándares no lo hacen" (las traducciones son mías).
} 
cial y cultural en donde la posible separación no tiene lugar (Hom y May, 2018): en concreto, sus análisis coinciden en que el impacto del uso de un slur en las condiciones de verdad de un enunciado es pleno, y acarrea consecuencias directas en el análisis y la evaluación.

Estas dos son las posturas principales, pero a partir de ellas han surgido versiones matizadas. Una de particular relevancia y atractivo es la de Díaz Legaspe (2018), que disecciona las tesis principales de los argumentos centrales de ambas posturas para explicar así una taxonomía basada en el alcance y tipo de referencia de los slurs. Captura así la segunda consideración expuesta previamente, en tanto presenta un modelo operativo de clasificación de dos tendencias.

En el presente trabajo se expondrá el sistema de Díaz Legaspe, basado en la restricción referencial, para dar cuenta de que esta propuesta es compatible con el marco de análisis de la semántica situacional. Las consideraciones teóricas esbozadas por Barwise y Perry en su libro fundacional, Situation and Attitudes (1983), contemplan mecanismos que actúan de manera propicia para complementar la propuesta de Díaz Legaspe, ya que mantienen los criterios de aplicación de cada uno de los tipos.

\section{Tesis de la contraparte neutra}

Desde Kaplan (1999), la posibilidad de que una parcialidad del significado de determinados términos formara parte de una nueva dimensión semántica cobró fuerza en los estudios sobre expresividad. Fue particularmente influyente en las teorías neogriceanas que se focalizaron en sofisticar la noción de implicatura convencional, a saber: Potts (2005, 2007), McCready (2010), Gutzmann (2015), entre otros.

El tratamiento que han recibido los términos peyorativos dentro de esta corriente ha sido consistente. Lo que se sostiene desde Grice (1975) es que en el caso de las implicaturas convencionales existe un significado que no es eminentemente pragmático ni veritativo-funcional, pero que está asociado al significado convencional del término. Esta doble contribución se tradujo en un modelo de análisis de dos dimensiones: una correspondiente al significado veritativo-funcional, y la otra al expresivo o convencionalmente 
implicaturado. La primera de ellas es la ya aludida contraparte neutra, cuya función e importancia ocupa un lugar central en las propuestas de análisis de slurs.

Díaz Legaspe propugna dos tesis para sintetizar el peso de la contraparte neutra en las dos corrientes principales:

[Application Neutral Counterpart Thesis]: For every slurring expression $e$ there is an (actual or potential) neutral counterpart $\mathrm{NC}_{\mathrm{e}}$ such that $\mathrm{NC}_{\mathrm{e}}$ 's correct application criteria are identical to $e$ 's correct application criteria.

[Referential Neutral Counterpart Thesis]: For every slurring expression $e$ there is an (actual or potential) neutral counterpart $\mathrm{NC}_{\mathrm{e}}$ such that the class of individuals referred to by $\mathrm{NC}_{\mathrm{e}}$ 's (call it $\{\mathrm{NCe}\}$ ) is identical to the class of individuals referred to by $e$. (DÍAZ LEGASPE, 2018, p.2) ${ }^{4}$

La primera de ellas (en adelante, ANCT) le otorga una importancia moderada: lo que comparte el slur con una contraparte potencial o real son únicamente sus condiciones de aplicación. La segunda (en adelante, RNCT), en cambio, sostiene que el solapamiento se extiende incluso a la referencia. En concreto, el slur y su contraparte neutra son veritativo-funcionalmente intercambiables: lo único que aporta el uso de un slur es un matiz expresivo, una variación en la fuerza peyorativa. Esta fuerza peyorativa es la carga semántica inherente al slur según RNCT, que se condice con el comportamiento en la descripción general de slur de Cepollaro y Thommen expuesta previamente.

ANCT es universalmente aceptada. Según Díaz Legaspe, captura la intuición básica de que usar un slur dirigido hacia una o varias personas que no pertenezcan a la clase referencial comprendida por la contraparte neutra cuenta como una falla de competencia lingüística o epistémica. RNCT, por su parte, ha presentado una serie de problemas, de los cuales se detallarán dos a continuación.

En primer lugar, existen casos en los que la sustitución de un slur por su contraparte neutra resulta contraintuitiva o problemática. Un ejemplo

\footnotetext{
4 “Tesis de la Aplicación de la Contraparte Neutra: Para toda expresión slur $e$ hay una contraparte neutra (real o potencial) $\mathrm{NC}_{\mathrm{e}}$ tal que los criterios de aplicación correcta de $\mathrm{NC}_{\mathrm{e}}$ son idénticos a los criterios de aplicación correcta de $e$.

Tesis de la Referencia de la Contraparte Neutra: Para toda expresión slur $e$ hay una contraparte neutra (real o potencial) $\mathrm{NC}_{\mathrm{e}}$ tal que la clase de individuos a los que se refiere con $\mathrm{NC}_{\mathrm{e}}$ (llámese $\left\{\mathrm{NC}_{\mathrm{e}}\right\}$ ) es idéntica a la clase de individuos a la que se refiere con $e$."
} 
clásico que valida esta visión es una sentencia extraída de un monólogo del comediante Chris Rock:

(1) I love black people, but I hate niggers.

Amo a la gente negra, pero odio a los negros.

Si aquí reemplazáramos el slur por su contraparte neutra, el resultado sería un enunciado contradictorio. Las condiciones de verdad de este enunciado reformulado definitivamente no serían equivalentes a las del enunciado original. Llevado hasta las últimas consecuencias, incluso ANCT fallaría: los criterios de aplicación de las dos expresiones parecen diferir. Lo que apunta Díaz Legaspe — que resulta más evidente aún cuando se considera el monólogo completo- es que el grupo target del slur no es concomitante con la clase referencial a la que se asocia normalmente, sino que se trata de una porción menor a su interior. Es decir, si consideramos esa clase referencial como un conjunto, en el ejemplo de Chris Rock el slur estaría dirigido a un subconjunto dentro de esa clase. A su vez, dadas las condiciones de esta emisión, se puede observar que el uso de the $N$-word es más cercano a un uso figurativo que a un uso literal, lo que de hecho refuerza la intención de seleccionar un sub-grupo del grupo target, estrechamente ligado al efecto cómico al que pueda dar lugar.

En segundo lugar, Ashwell (2016) ha presentado algunos casos de slurs en los cuales es difícil o imposible hallar una contraparte neutra. En especial, este escollo resulta saliente en los slurs vinculados a la discriminación por género. Bitch, sissy y slut son los ejemplos que ella analiza con más detenimiento: parafrasear cada uno de ellos por términos derogatoria y normativamente neutrales y equivalentes es una tarea condenada al fracaso. ANCT, estima Díaz Legaspe, puede aplicarse en su articulación negativa (solo las mujeres podrían ser llamadas puta $-\mathrm{y}$, en el ejemplo de Chris Rock, solo los afroamericanos podrían ser llamados the $N$-word-), pero no a la inversa, en su articulación positiva (no es verdadero que todas las mujeres podrían ser correctamente llamadas puta, ni — como expresa el mismo ejemplo - tampoco es verdadero que se pueda llamar correctamente a todos los afroamericanos por the $N$-word).

Tras demostrar que, según su visión, la propuesta de la contraparte nuestra resulta insuficiente para explicar estos casos, Ashwell postula una 
nueva manera de contemplar el fenómeno de los slurs en su conjunto: lo que se encuentra a la base del criterio de aplicación no es una paráfrasis menos expresiva, sino una o varias normas o estándares sociales impuestos sobre el grupo target. La fuerza derogatoria, entonces, surge del incumplimiento o la desviación por parte de ese grupo con respecto a esa(s) norma(s) que el hablante usuario del slur le aplica al target. El slur evidencia una sanción social o la marginalización de un grupo cuyo comportamiento no coincide con lo que se espera de él.

Del señalamiento de la aplicación positiva y negativa de ANCT se desprende que la consideración de Díaz Legaspe de este segundo problema es similar a la del primero: más allá del elemento que defina al grupo target - la co-rreferencia total o el comportamiento "inadecuado"-, subsiste el hecho de que la aplicación del slur se realiza correctamente sobre un subgrupo cuantitativamente menor a la clase referencial general a la que podría hacer referencia. Según ella, lo que distingue a estos slurs de aquellos en los que resulta más sencillo encontrar una contraparte neutra (por ejemplo, los de tipo demográfico, como bolita o sudaca) es, primordialmente, una restricción referencial: el hecho de que el slur refiera a un subconjunto de la clase referencial.

\section{La taxonomía de Díaz Legaspe}

En la sección anterior se repusieron las dos tesis sobre las que descansan las principales corrientes de análisis de los slurs, conjuntamente a dos posibles problemas que emergen en su aplicación a casos específicos. Hacia el final se mencionó que, más allá de estos casos, la generalidad de los slurs que suelen ser objeto de análisis de los defensores de RNCT no presentan las mismas complicaciones: cuando el grupo target está delimitado, por ejemplo, por su localización demográfica, lo habitual es que las contrapartes neutras exhiban los mismos criterios de aplicación que los slurs. Bolita se aplicará correctamente en los casos en que el hablante que lo emita sienta desprecio por una persona nacida en Bolivia, es decir, por un/a boliviano/a. 
Frente a esta multiplicidad de casos y sus particularidades, se impone una toma de decisión metodológica. Como se describió en la introducción, incluso las caracterizaciones mismas de qué es un slur y qué no lo es difieren entre teorías y autores, por lo que una de las posibles estrategias podría consistir en priorizar un tipo y explicar el otro en términos de otro fenómeno. Sin embargo, perviven a través de todos los ejemplos las condiciones básicas puestas en palabras de Cepollaro y Thommen: expresan una fuerza peyorativa hacia grupos con propiedades específicas, y suscitan reacciones emocionales fuertes entre quienes no los usan. Lo más adecuado parece, entonces, asumir, más que una distinción ontológica, una clasificación de distintos tipos de slurs al interior de la clase.

Esta es la tarea que emprende Díaz Legaspe. A grandes rasgos, postula dos tipos básicos: por un lado, los slurs normalizantes (normalizing) y, por el otro, los slurs "marginales" (out-group). La diferencia central entre ellos es la opcionalidad o no de la restricción referencial: en los normalizantes (puta, maricón, etc.) esta restricción es necesaria, mientras que en los "marginales" (bolita, villero, etc.) es meramente opcional. La intención de base es la de dar cuenta de los casos de Ashwell poniendo de manifiesto las particularidades que ella misma halló ${ }^{5}$, aunque Díaz Legaspe conserve los criterios más extendidos para la identificación y el análisis de los slurs.

Que para los slurs normalizantes la restricción referencial no sea opcional quiere decir que en todos los casos en que se usen referirán obligatoriamente a un subgrupo dentro de una clase más extendida. Los "marginales", en cambio, pueden referir a un conjunto entero asociado a determinada clase (como es el caso de bolita = boliviano, discutido más arri-

\footnotetext{
5 Un paso relevante en esta dirección es un aspecto sobre el que todavía no se ha hecho hincapié pero que está comprendido en las mismas definiciones de RNCT y ANCT. Díaz Legaspe introduce una sutileza en cuanto a la contraparte neutra que aliviana el peso por el que Ashwell descarta ese análisis: "real o potencial", "even if it is not available yet, or the wrong choice of neutral candidate has been made, were the potential or correct candidate to be found, the slur would be co-referential with it" (2018:4) ["incluso si todavía no estuviera disponible, o se ha elegido incorrectamente un candidato neutro, si el candidato potencial o correcto todavía estuviera por hallarse, el slur sería correferencial con respecto a él']. Incluso explicita que es un tanto contraintuitivo considerar algún caso de slur en el que no haya al menos un candidato potencial a ser su contraparte. Esto es particularmente relevante porque es esa misma contraparte neutra una herramienta fundamental para determinar cuál será la referencia en cada caso, y aún más cuando se trate de casos en los que haya restricción referencial.
} 
ba), pero también pueden seleccionar un subconjunto al interior de esa clase como grupo target: este es el caso del ejemplo de Chris Rock.

Para que su marco de análisis resulte más operativo a la hora de explicar esta última variación entre slurs marginales, Díaz Legaspe hace una distinción en la implementación de la restricción referencial: hay tanto significados con restricción referencial como usos con restricción referencial. Los primeros se identifican con los slurs normalizantes, y los segundos serían los casos en los que un slur marginal se usa para referir a un subgrupo. Sin embargo, lo cierto es que palabras que no se asocian regularmente con slurs pueden también tener usos referencialmente restringidos que apunten a expresar peyoración o desprecio por un subgrupo particular. Considérese el caso de los primeros versos de la canción "Loca" del cantante de trap argentino Khea, hecha en colaboración con Duki y Cazzu:

(2) Es una loca, yeah

Me manda videos al Snap mientras se toca, yeah

Me dice que si hoy le llego, que no puede esperar, yeah

Que se muere por mí, que quiere to' conmigo

Que la vaya a buscar, yeah, que la vaya a buscar

La expresión loca, en el primer verso, no está queriendo significar que la mujer de la que se habla es necesariamente una persona que sufre de locura o que no actúa en su sano juicio, que es el significado más usual que suele tener el término. Por el contrario, aquí parece ser más cercano a lo que podría ser una contraparte neutra potencial para términos como puta, parafraseable en mayor o menor medida por "mujer promiscua" (cuanto menos, "mujer que no se comporta de acuerdo a las expectativas sexoafectivas que recaen sobre ella"). Este nuevo significado es mucho más transparente teniendo en cuenta los versos siguientes: el cantante pone de relieve una serie de comportamientos por las cuales le atribuye a esta mujer semejante calificativo. Por lo tanto, loca, en este contexto, está constituyendo un uso restringido referencialmente. El hablante tiene la intención de categorizar peyorativamente a un conjunto de mujeres que exhiben un tipo determinado 
de comportamiento que se desvía de una norma social y/o cultural preestablecida ${ }^{6}$.

La taxonomía de Díaz Legaspe queda, entonces, definida de la siguiente manera: hay dos tipos de slurs, normalizantes y marginales; en el primero de ellos la restricción referencial es obligatoria, y en el segundo no. De esta distinción nace una segunda, del orden de la estrategia discursiva, que contempla la restricción referencial en dos modalidades diferentes: significados (donde es constitutiva e inherente al término la selección de un subgrupo dentro de una clase) y usos (donde la referencia parcial es producto de una intención comunicativa encriptada en un término que suele tener una referencia más amplia).

\section{La semántica situacional}

Al introducir la noción de restricción referencial, Díaz Legaspe realiza una salvedad. Hay dos maneras posibles de explicar este fenómeno: en primer lugar, apelar a la sensibilidad contextual de los slurs y clasificarlos como una suerte de indéxicos, variando en cada contexto la contribución veritativo-funcional; en segundo lugar, que la correferencialidad dependa enteramente de usos particulares y sus contrapartes, permitiendo que no sea el slur per se el que correfiera siempre con su contraparte neutra, sino que la correferencialidad se dé únicamente en el uso. Al respecto, Díaz Legaspe enuncia lo siguiente:

Both options are good alternatives; they are even compatible with the solution offered in this paper to the problem of restrictive reference in particular uses of a certain type of slurs (demographic, outgroup slurs), in which a term of this type is used to refer to a subset of a larger class. However, these alternatives fail to explain two points: first, an explanation is owed for the fact that even in these cases, the standard, typical use of this type of slurs is strongly

\footnotetext{
6 Todos ejemplos aquí brindados de slurs normalizantes (tanto en el plano del significado como en el plano del uso, como es este caso) son términos peyorativos en razón del desprecio por las disidencias de género, lo cual no resulta llamativo si se tiene en cuenta que una de las características principales de este tipo de slurs es una desviación con respecto a una o varias normas sociales y/o culturales que muchas veces se condicen con los valores y principios de sociedades y culturas heteronormadas y patriarcales. Ahora bien, esta selección no pretende establecer que todos los slurs normalizantes son slurs de género: considérense otros términos peyorativos que nacen de la desviación de normas sociales - como cheto o cabeza (corrientes en el español rioplatense) - y el análisis y clasificación valdrá también para ellos.
} 
linked to particular neutral counterparts, which makes referentially restrictive uses a deviation. Second, when it comes to gendered (normalizing) slurs, there is no context nor use in which the slur is co-referential with the associated neutral counterpart; the pervasiveness of this referential restriction should be also explained (DÍAZ LEGASPE, 2018, p.4)7.

El propósito de este apartado es señalar que una de las vías compatibles semánticamente con su propuesta es la de la semántica de situaciones $\mathrm{y}$, al mismo tiempo, dar cuenta de que las dos fallas que Díaz Legaspe apunta a propósito de una solución contextualista —a saber, la falta de explicación de los casos en los que la restricción referencial es opcional, y la no co-referencialidad con una contraparte neutra en los slurs normalizantes - pueden encontrar reparo bajo este marco de análisis.

La semántica situacional nació en la década de 1980, de la mano de un matemático, Jon Barwise, y un filósofo, John Perry. La motivación central fue brindar una alternativa al modelo de mundos posibles, con un afán de sofistificación, en tanto las bases para la evaluación de los enunciados no fueran mundos completos sino parcialidades de mundos. Eso son las situaciones: básicamente, la tesis principal que defiende esta teoría es que la gente usa el lenguaje en partes limitadas del mundo para hablar de partes limitadas del mundo. Hay un claro énfasis en la parcialidad que distingue a la semántica situacional de su predecesora, la semántica de mundos posibles: "[s]ituations are parts of the world and the information an agent has about a given situation at any moment will be just a part of all the information that is theoretically available" (DEVLIN, 2006, p.2). A fin de cuentas, la semántica situacional es una teoría relacional del significado: "meaning in general, and

\footnotetext{
7 "Ambas opciones son buenas alternativas; incluso son compatibles con la solución ofrecida en este artículo al problema de referencia restrictiva en usos particulares de un tipo particular de slurs (demográficos, slurs marginales), en los que un término de este tipo se usa para referir a un subconjunto de una clase más amplia. Sin embargo, estas alternativas fallan a la hora de explicar dos puntos: primero, se necesita una explicación para el hecho de que incluso en estos casos, el uso estándar, típico de este tipo de slurs está fuertemente ligado a contrapartes neutras particulares, lo que convierte a los usos referencialmente restrictivos en una desviación. Segundo, cuando se considera el caso de los slurs ligados al género (normalizantes), no hay ningún contexto ni uso en el que el slur sea co-referencial con respecto a su contraparte neutra; la omnipresencia de esta restricción referencial también debería ser explicada".

8 "Las situaciones son partes del mundo y la información que un agente tiene acerca de una situación dada en cualquier momento será solamente una parte de toda la información que está teóricamente disponible".
} 
linguistic meaning in particular, is a relation between situations"9 (BARWISE; PERRY, 1983, p.6).

El objeto de estudio por excelencia de la semántica situacional son las emisiones. En cada emisión, pueden identificarse tres situaciones básicas: i) la situación de emisión (el contexto inmediato, en el que la emisión se produce y se recibe), ii) la situación de referencia (tanto la percepción del hablante como la manera en la que es el mundo, el conocimiento común acerca de él, y el discurso previo) y iii) la situación focal (la situación descripta en el material lingüístico, la parte del mundo sobre la que es la emisión). Podríamos traducir este esquema tríptico en uno de círculos concéntricos, en el que el centro fuera la situación focal, la segunda capa la situación de referencia, y la tercera la situación de emisión.

De lo dicho previamente se desprende que, si la semántica situacional es una teoría relacional del significado, estas tres situaciones básicas necesariamente interactúan entre sí, y el significado, en cada emisión, dependerá de su interrelación. Con este esquema básico en mente, podemos pasar a examinar cómo este modelo puede dar cuenta de los tipos de slurs propuestos por Díaz Legaspe.

En lo que concierne a los slurs normalizantes — la segunda objeción - , una de las primeras ventajas a tener en cuenta es que, al tomar para la evaluación solamente una parcialidad de mundo, la semántica situacional da respaldo a dos aspectos centrales de la sensiblidad contextual: por un lado, no es incongruente que, dado que en determinada parcialidad de mundo estén vigentes ciertas normas sociales y/o culturales que en otra parcialidad pueden no estarlo, haya distintas parcialidades del mundo (real) en las que un slur sea de hecho un slur y en otras parcialidades no lo sea ${ }^{10}$. Por otro lado - y esta consideración es extensible a todos los slurs en su conjunto-, incluso es propicio el énfasis en la parcialidad para explicar la variación dia-

\footnotetext{
9 “el significado en general, y el significado lingüístico en particular, es una relación entre situaciones".

${ }^{10}$ Considérese el caso de the $N$-word previamente discutida: en Argentina, actualmente, la palabra negro para referirse a una persona no necesariamente codifica un significado peyorativo. Puede, incluso, ser muestra de afecto y cercanía por parte del hablante con respecto a su interlocutor.
} 
crónica del peso derogatorio de los slurs: existen términos que han sido slurs y dejaron de serlo, así como también ocurre a la inversa ${ }^{11}$.

Lo central es que la situación de referencia, en todos los casos de emisiones de slurs, actúa directamente en la determinación de la situación focal, ya que preserva las normas sociales y/o culturales vigentes en cuya violación se funda su significado. Es la situación de referencia —en última instancia - la que brinda, para tender el paralelismo entre las dos propuestas, las condiciones de aplicación correcta del slur, y la que interviene en la selección de la situación focal.

En cuanto a la correferencialidad directa con una contraparte neutra, en su explicación de RNCT y ANCT Díaz Legaspe expone las razones por las cuales no es necesario en su modelo que la contraparte sea real, sino que puede tratarse de un candidato potencial: es difícil — como sucedió en el análisis de "Loca"- no considerar al menos un candidato potencial que opere como contraparte. A su vez, la vigencia de las normas en el contexto dado puede facilitar aún más la emergencia de un candidato potencial para el slur ${ }^{12}$.

La primera de las objeciones apunta al caso contrario: qué sucede con los slurs marginales, que son más estables en su aplicación y selección referencial. La respuesta, estructuralmente, es la misma: es la situación referencial la que va a determinar, más consistentemente, la recurrencia de la situación focal. Una posible explicación corre por cuenta de la flexibilidad de esta segunda capa: si los slurs demográficos son menos restringidos referencialmente, en un marco situacional esto podría traducirse en una especificación menor en el nivel de la situación de referencia. Es decir: las condiciones de aplicación de los slurs normalizantes están más especificadas (lo cual hace sistema con la violación de normas vigentes en una parcialidad de mundo determinada) que las de los slurs marginales; el peso en la

\footnotetext{
${ }^{11}$ Un caso de aminoración en la fuerza peyorativa es negro (que, dadas las condiciones sociopolíticas a lo largo de la historia argentina, es más próximo a un slur normalizante — en tanto el desprecio no era debido al color de la piel, sino a la desviación con respecto a una norma social-), pero también lo es tano, que solía ser un slur demográfico dirigido a los inmigrantes italianos, y hoy es meramente una expresión coloquial con la misma referencia sin carga derogatoria.

12 Incluso, desde un análisis similar al que hace Potts (2007) de las implicaturas convencionales, cabría la posibilidad de estipular que las contrapartes neutras no son necesariamente lingüísticas, en cuyo caso podría tratarse de una serie de rasgos que, dando por hecho su dinamicidad, se saturarían contextualmente.
} 
determinación de las condiciones de aplicación no está tan acentuado por la vinculación con las normas vigentes, pero sí con las representaciones vigentes (temporal y espacialmente) en el contexto de emisión.

Por último, cabe mencionar que la semántica situacional no solo armoniza con la taxonomía general de Díaz Legaspe, sino que también contempla la distinción entre uso y significado, ambos pasibles de ser restrictivos referencialmente. Para todo término, existe, en este marco, un significado abstracto que no es sensible al contexto, y que responde, en esencia, a la pregunta “¿qué significa esta construcción en general?”. Empero, a un tiempo, hay un segundo significado, el significado en-uso, que vincula una emisión particular con el hecho en el mundo que es de la manera que se expresa en la emisión: responde a la pregunta "¿qué significa esta construcción como está usada en esta instancia?" Así, las restricciones referenciales mantienen la posibilidad de implementarse en un nivel general como en uno particular, tal como estipula Díaz Legaspe.

\section{Conclusiones}

Los argumentos expuestos hasta aquí permiten afirmar que el modelo clasificatorio de Díaz Legaspe encuentra fundamento semántico en el modelo de la semántica situacional. La piedra de toque de su programa — la restricción referencial - no pierde fuerza ni validez en el análisis situacional; por el contrario: la estructura de situaciones contribuye en la determinación de la referencia, sosteniendo una versión de RNCT con dependendencia fuerte de ANCT. Queda para futuros trabajos especificar analíticamente la manera en que esta interacción entre los distintos niveles de situaciones ocurre.

\section{Referencias}

ASHWELL, Lauren. Gendered Slurs. Social Theory and Practice 42 (2): 228-239, 2016.

BARWISE, Jon; PERRY, John. Situations and Attitudes. Cambridge/Mass.: The MIT Press, 1983. 
CEPOLLARO, Bianca; THOMMEN, Tristan. What's wrong with truth-conditional account of slurs. Linguistics and Philosophy, Vol. 42, 333-347, 2019.

DEVLIN, Keith. Situation theory and situation semantics. In: GABBAY, D.; WOODS, John (eds.) Handbook of the History of Logic, Vol. 7, 601-664, 2006 .

DÍAZ LEGASPE, Justina. Normalizing Slurs and Out-group Slurs: The Case of Referential Slurs. Analytic Philosophy, Vol. 59 No. 2, pp. 1-22, 2018.

GRICE, Herbert Paul. Logic and conversation. In: COLE, P.; MORGAN, J. (eds.), Syntax and semantics III: Speech acts, pp. 41-58. Nueva York: Academic Press, 1975.

GUTZMANN, Daniel. Use Conditional Meaning. Studies in Multidimensional Semantics. Oxford: Oxford University Press, 2015.

HOM, Cristopher; MAY, Robert. Pejoratives as Fiction. In: SOSA, D. (ed.). Bad Words. Oxford: Oxford University Press, 2018.

KAPLAN, David. The meaning of ouch and oops: Explorations in the theory of meaning as use. MS, University of California, Los Angeles, 1999.

MCCREADY, Elin. Varieties of conventional implicature. Semantics \& Pragmatics, Vol. 3, 1-57, 2010.

POTTS, Christopher. The logic of conventional implicature. Oxford: Oxford University Press, 2005.

. Into the Conventional-Implicature Dimension. Philosophy Compass, 2: 665-679. doi: 10.1111/j.1747-9991.2007.00089.x, 2007. 